\title{
Filocrono em diferentes densidades de plantas de batata
}

\author{
Phyllochron at different plant densities in potato
}

\author{
Jacso Dellai $^{1}$ Gustavo Trentin ${ }^{1}$ Dilson Antônio Bisognin ${ }^{2}$ \\ Nereu Augusto Streck ${ }^{2}$
}

\section{RESUMO}

O conceito do filocrono, ou seja, o intervalo de tempo necessário para a emissão de duas folhas consecutivas, pode ser usado para simular o aparecimento de folhas em plantas. $O$ número de folhas, além de afetar o índice de área foliar da planta, é considerado uma excelente medida de tempo fisiológico. O objetivo do trabalho foi estimar o filocrono em batata cultivada num sistema hidropônico com substrato em diferentes densidades de plantas. $O$ experimento foi conduzido em ambiente protegido, no Departamento de Fitotecnia da UFSM, RS, no período de 03/09/2004 a 26/10/ 2004. O delineamento experimental foi o inteiramente casualizado, com três repetições. Os tratamentos foram os espaçamentos de 5x5, 10x10,15x15 e 20x20cm entre covas. Os valores de filocrono para cada densidade foram relacionados com o número de covas $\mathrm{m}^{-2}$ e de hastes $\mathrm{m}^{-2}$. Os valores de filocrono estimados variaram em função da densidade de plantas. As maiores densidades promoveram aumentos no filocrono, indicando que a densidade de plantas afeta a velocidade de surgimento de folhas em batata, e esta resposta deve ser contemplada em modelos de simulação do número de folhas nesta cultura.

Palavras-chave: Solanum tuberosum L., soma térmica, emissão de folhas, competição intraespecífica.

\section{ABSTRACT}

The phyllochron, defined as the time interval between the appearance of successives leaves, can be used to simulate leaf appearance in plants. The number of leaves affects the leaf area index of the plant and is considered an excellent measure of physiological time. The objective of this work was to estimate the phyllochron in potato plants cultivated in hydroponic system with substrate in different plant densities. The experiment was carried out in a protected environment at the Department of Fitotecnia, UFSM, RS, from 03/09/2004 to 26/10/2004. The experimental design was completely randomized, with three repetitions. The treatments were the densities of $5 \times 5,10 \times 10,15 \times 15$ and $20 \times 20 \mathrm{~cm}$ between hills. The phyllochron values for each density were related to the number of hills $\mathrm{m}^{-2}$ and stems $\mathrm{m}^{-2}$. The estimated phyllochron values varied according to plant density. Phyllochron increased with plant density, showing that plant density affects the leaf appearance rate in potato, and this response should be taken into account in the simulation models of the number of leaves in this crop.

Key words: Solanum tuberosum L., thermal time, leaf emergence, intra-specific competition.

\section{INTRODUÇÃO}

A batata (Solanum tuberosum L.) ocupa o quarto lugar em volume de produção mundial de alimentos (299 milhões de toneladas), sendo superada pelo trigo (595 milhões de toneladas), milho (592 milhões de toneladas) e arroz (571 milhões de toneladas) (FAO, 1998). O cultivo de batata no Brasil foi intensificado na década de 20, sendo hoje considerada a principal hortaliça no país, tanto em área cultivada (cerca de 170.000 ha ano $^{-1}$ ) como em

\footnotetext{
${ }^{1}$ Programa de Pós-graduação em Agronomia, Universidade Federal de Santa Maria (UFSM), 97105-900, Santa Maria, RS, Brasil. ${ }^{2}$ Departamento de Fitotecnia, Centro de Ciências Rurais, UFSM, 97105-900, Santa Maria, RS, Brasil. E-mail: nstreck1@smail.ufsm.br. Autor para correspondência.
} 
preferência alimentar (LOPES \& BUSO, 1997). A produção de batata concentra-se nos Estados de Minas Gerais, São Paulo, Paraná e Rio Grande do Sul, sendo estes Estados responsáveis por aproximadamente $98 \%$ da produção nacional (IBGE, 2004).

A qualidade da batata-semente é um dos principais fatores que afetam a produtividade e qualidade da cultura da batata (KIM et al., 2000). Mesmo que as demais condições sejam favoráveis ao cultivo, o plantio de tubérculos-semente de baixa qualidade pode comprometer a produtividade de uma safra. A produção de batata-semente pré-básica em sistema hidropônico mostra-se como uma tecnologia com excelente potencial, quando se busca a produção de batata-semente de alta qualidade fitossanitária. O sistema hidropônico é muito vantajoso para a produção de batata pré-básica pela melhoria da produtividade e da sanidade (MEDEIROS et al., 2002). Esta é uma tecnologia recente, e questões básicas de manejo, como a densidade de plantas, precisam ser entendidas para aumentar a eficácia do sistema.

O número de folhas acumulados em uma haste é uma excelente medida de tempo fisiológico e está associado ao momento de ocorrência de estágios de desenvolvimento e ao início da ramificação lateral em diversas espécies vegetais (STRECK et al., 2003), incluindo a batata (VOS \& BIEMOND, 1992). A velocidade de emissão de folhas também influencia a evolução do índice de área foliar da planta. Uma maneira de simular o aparecimento de folhas na haste principal de plantas é através do conceito do filocrono, definido como o intervalo de tempo necessário para a emissão de folhas consecutivas (KLEPPER et. al, 1982; FRANK \& BAUER, 1995; HERMES et. al., 2001). O método da soma térmica tem sido freqüentemente usado para representar o efeito da temperatura do ar sobre o crescimento e desenvolvimento das plantas (JEFFERIES, et al., 1987), caracterizando-se por ser um método simples e uma melhor medida de tempo fisiológico do que dias do calendário civil ou dias após a semeadura (STRECK, 2002). Utilizando-se a soma térmica como medida de tempo fisiológico em plantas, o filocrono corresponde aos graus-dia necessários para o aparecimento de uma folha $\left({ }^{\circ} \mathrm{C}\right.$ dia folha $\left.{ }^{-1}\right)$.

Não estão disponíveis na literatura informações referentes à velocidade de surgimento de folhas em batata cultivada em diferentes densidades. É possível que a densidade de plantas altere a taxa de surgimento de folhas, fazendo com que a densidade de plantas tenha que ser considerada em modelos de simulação do aparecimento de folhas em batata. O objetivo deste estudo foi estimar o filocrono em batata cultivada em substrato em diferentes densidades de plantas.

\section{MATERIAL E MÉTODOS}

O experimento foi conduzido em telado do Programa de Genética e Melhoramento de Batata, Departamento de Fitotecnia da UFSM. O cultivo das plantas foi feito em substrato de areia média que recebia quatro sub-irrigações diárias com solução nutritiva (ANDRIOLO \& POERSCHKE, 1997) num sistema hidropônico fechado. Cada canteiro continha 1,10m de largura, 3,80m de comprimento e 1,5\% de declividade.

Os tratamentos consistiram de diferentes espaçamentos de plantio de tubérculos de batata, cv. Macaca. Cada tratamento foi disposto em um canteiro nos espaçamentos entre tubérculos de: $5 \times 5 \mathrm{~cm}$ (D1: 400covas $\mathrm{m}^{-2}$ ), 10x10cm (D2: 100covas $\left.\mathrm{m}^{-2}\right), 15 \times 15 \mathrm{~cm}$ (D3: 44covas $\mathrm{m}^{-2}$ ) e $20 \mathrm{x} 20 \mathrm{~cm}$ (D4: 25 covas $\mathrm{m}^{-2}$ ). O delineamento experimental utilizado foi o inteiramente casualizado com três repetições.

O plantio dos tubérculos foi efetuado no dia 03/09/2004. Logo após a emergência, foram marcadas três plantas em cada repetição na parte central, deixando-se uma linha de plantas como bordadura, perfazendo um total de nove plantas em cada tratamento. Nessas plantas, foi contado o número de folhas na haste principal, duas vezes por semana, até o final do período de emissão de folhas, correspondente ao dia 26/10/2004. Foi considerado como folha visível quando o folíolo apical tinha pelo menos $1 \mathrm{~cm}$ de comprimento (CAO \& TIBBITTS, 1995). Nestas plantas, também foi medido o comprimento da haste principal, nas mesmas datas em que se realizou a contagem do número de folhas. Além do número de covas $\mathrm{m}^{-2}$, também foi determinado, no final do experimento, o número médio de hastes em 10 covas de cada repetição.

A temperatura do ar no interior do telado foi medida com o auxílio de um termohigrógrafo de registro semanal, instalado no interior de um miniabrigo de madeira pintado de branco, a 1,5m acima do nível dos canteiros. Os valores de temperatura foram coletados em intervalos de duas horas. Através destes dados, determinou-se a temperatura média diária $\left(\mathrm{T}_{\mathrm{m}}\right)$ somando-se os valores de temperatura coletados e dividindo-se pelo número de observações:

$\mathrm{T}_{\mathrm{m}}=\left(\mathrm{T}_{0 \mathrm{~h}}+\mathrm{T}_{2 \mathrm{~h}}+\ldots+\mathrm{T}_{20 \mathrm{~h}}+\mathrm{T}_{22 \mathrm{~h}}\right) / 12$

A soma térmica diária (STd) foi calculada pelo seguinte método: 
$\operatorname{STd}=\left(\mathrm{T}_{\mathrm{ot}}-\mathrm{T}_{\mathrm{b}}\right) \cdot\left(\mathrm{T}_{\mathrm{m}}-\mathrm{T}_{\mathrm{b}}\right) /\left(\mathrm{T}_{\mathrm{ot}}-\mathrm{T}_{\mathrm{b}}\right)$, quando $\mathrm{T}_{\mathrm{b}} \leq \mathrm{T}_{\mathrm{m}} \leq$ $\mathrm{T}_{\mathrm{ot}}$

STd $=\left(T_{o t}-T_{b}\right) \cdot\left(T_{\max }-T_{m}\right) /\left(T_{\max }-T_{o t}\right)$, quando $T_{o t}<$ $\mathrm{T}_{\mathrm{m}} \leq \mathrm{T}_{\max }$

STd $=0$, quando $\mathrm{T}_{\mathrm{b}}>\mathrm{T}_{\mathrm{m}}>\mathrm{T}_{\text {max }}$

onde: $\mathrm{T}_{\mathrm{b}}, \mathrm{T}_{\mathrm{ot}}$ e $\mathrm{T}_{\max }$ são as temperaturas cardinais (base, ótima e máximas, respectivamente) para a taxa de aparecimento de folhas em batata, as quais foram consideradas como 7, 21 e $30^{\circ} \mathrm{C}$, respectivamente (SANDS et al. 1979), e $T_{m}$ é a temperatura média do ar no interior do telado. A representação gráfica da concepção do método de cálculo da STd está na figura 1.

Foi feita uma regressão linear entre o número de folhas acumulados na haste principal e a soma térmica acumulada (STa) desde a data de início da contagem do número de folhas (24/09/2004), onde: $\mathrm{STa}=\Sigma \mathrm{STd}$

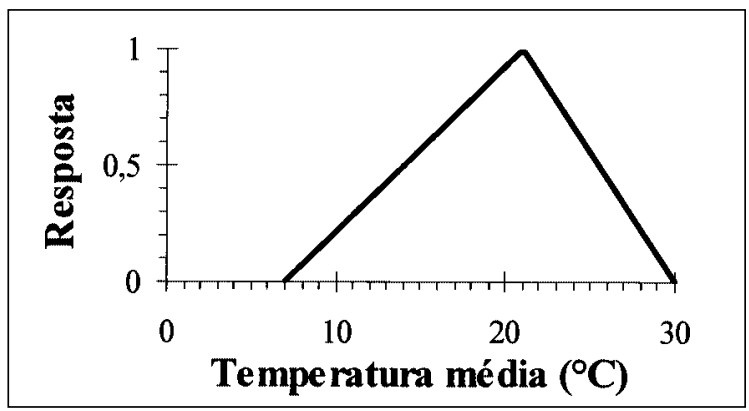

Figura 1 - Representação esquemática do método utilizado para o cálculo da soma térmica diária (STd).
O filocrono para cada densidade de plantas foi estimado pelo inverso do coeficiente angular da regressão linear (KLEPPER et. al, 1982). Os dados foram submetidos a análises de variância teste $F$, teste t e coeficiente de determinação em nível de $1 \%$ de probabilidade de erro, com o auxílio do software estatístico SAS (SAS Institute, 1997).

\section{RESULTADOS E DISCUSSÃO}

O número médio de hastes por cova nas diferentes densidades de plantio foi de 1,43, 1,83, 1,77 e 2,07 hastes, respectivamente, para as densidades D1, D2, D3 e D4. O aumento do número de hastes com a diminuição da densidade de covas pode ser explicado pela menor competição intra-específica nas densidades menores. Com os valores de espaçamento entre covas e o número médio de hastes por cova, foi possível estimar o número de covas $\mathrm{m}^{-2}$ e de hastes $\mathrm{m}^{-2}$ para cada tratamento, resultando em D1 $=400$ covas $\mathrm{m}^{-2} \mathrm{e}$ 573hastes $\mathrm{m}^{-2}$, D2 $=100$ covas $\mathrm{m}^{-2} \mathrm{e} 183$ hastes $\mathrm{m}^{-2}$, D3 $=44$ covas $\mathrm{m}^{-2}$ e 78hastes $\mathrm{m}^{-2}$ e D4=25covas $\mathrm{m}^{-2}$ e 52 hastes $\mathrm{m}^{-2}$.

Os valores absolutos de temperaturas mínima e máxima verificadas durante o experimento foram $6^{\circ} \mathrm{C}$ e $29^{\circ} \mathrm{C}$, respectivamente, sendo que a temperatura média durante todo o período foi de $16,1^{\circ} \mathrm{C}$. As temperaturas médias diárias do ar registradas no interior do telado estiveram quase sempre abaixo da $\mathrm{T}_{\mathrm{ot}}$ (Figura 2). Nas primeiras semanas após o início do experimento as temperaturas foram menores,

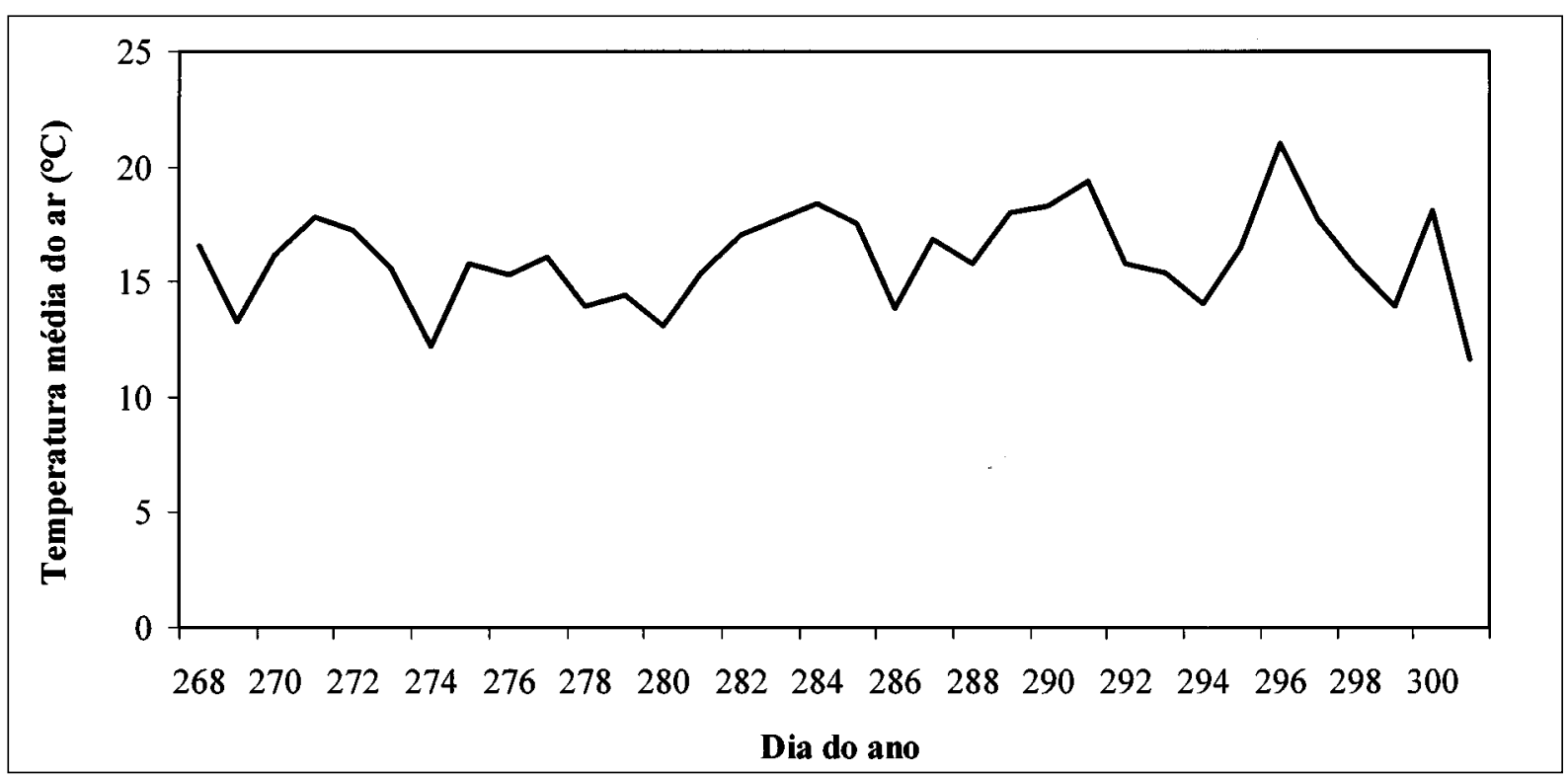

Figura 2 - Temperatura média diária do ar $\left({ }^{\circ} \mathrm{C}\right)$ registrada no interior do telado durante o período de 24/09/2004 a 27/10/2004. Santa Maria, RS, 2004.

Ciência Rural, v.35, n.6, nov-dez, 2005. 
aumentando no decorrer do ciclo da cultura. Esta variação pode ser explicada pelas condições de aumento do fotoperíodo, já que o aumento do número de horas de brilho solar pode ser relacionado com o aumento da temperatura (PEREIRA et al., 1997).

A relação entre o número de folhas na haste principal e a soma térmica acumulada foi linear, com um $\mathrm{R}^{2}>0,99$ para todos os tratamentos e o coeficiente angular significativo pelo teste t (Figura 3). Isso indica que a temperatura média do ar foi o fator ecológico principal que determinou a emissão de folhas em batata. Os valores de filocrono estimados variaram em função da densidade de plantas, sendo que o menor valor foi estimado na densidade $\mathrm{D} 4\left(19,2^{\circ} \mathrm{C}\right.$ dia folha $\left.{ }^{-1}\right)$ e o maior na densidade $\mathrm{D} 1\left(22,9^{\circ} \mathrm{C}\right.$ dia folha $\left.{ }^{-1}\right)$. As densidades D2 e D3 apresentaram valores de filocrono intermediários, ou seja, 20,9 e $20,2^{\circ} \mathrm{C}$ dia folha ${ }^{-1}$, respectivamente (Figura 3 ).

Foi ajustada uma função do tipo potencial para representar a relação entre o filocrono e o número de covas $\mathrm{m}^{-2}$ e de hastes $\mathrm{m}^{-2}$ (Figura 4). Optou-se pela função não-linear não apenas por apresentar um $\mathrm{R}^{2}$ mais alto do que a linear, mas também por certamente ser uma função mais realística do ponto de vista biológico, uma vez que a reta ajustada teria um valor de filocrono para uma densidade de zero covas $\mathrm{m}^{-2} \mathrm{ou}$ zero hastes $\mathrm{m}^{-2}$, o que não é realístico. Os resultados indicam a tendência do filocrono aumentar com o número de covas e/ou hastes por unidade de área, ou seja, as plantas cultivadas na densidade D1 demoram mais tempo para emitir folhas, desenvolvendo-se numa taxa menor, quando comparadas com as plantas das demais densidades. No entanto, ao se analisar o crescimento dessas plantas utilizando-se como parâmetro o comprimento da haste principal, verificouse que as plantas das densidades D1 e D2 apresentaram maior crescimento quando comparadas às demais. Essas densidades atingiram a maior estatura na última avaliação (Figura 5), diferindo das demais densidades significativamente pelo teste de Tukey a 5\%. Neste caso, as plantas das densidades D1 e D2 provavelmente destinaram maior parte dos fotoassimilados para a elongação da haste em detrimento da emissão de folhas, devido à maior competição intra-específica por luz (OLIVEIRA, 2000). Essa tendência oposta de resposta do filocrono e do

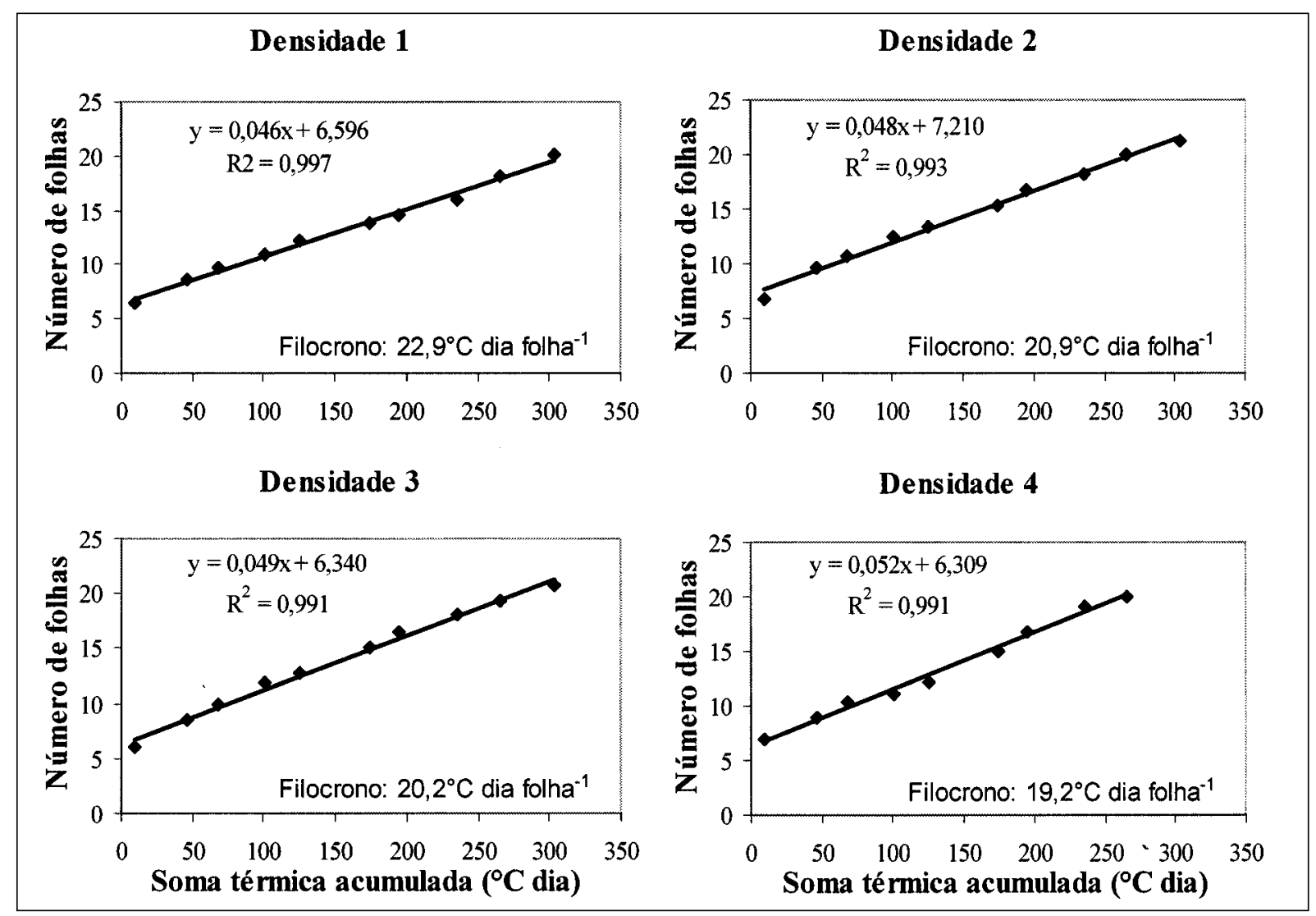

Figura 3 - Relação entre número de folhas acumuladas na haste principal e soma térmica acumulada utilizada para a estimativa do filocrono em batata, cv. Macaca. Santa Maria, RS, 2004. 

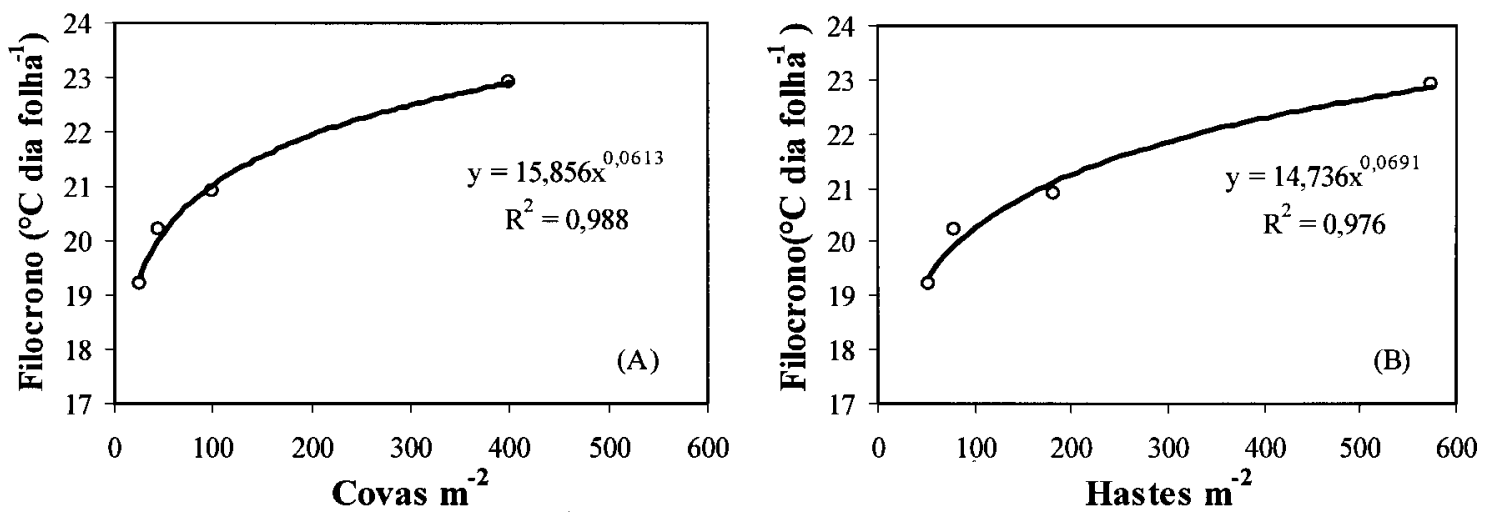

Figura 4 - Relação entre filocrono e número de covas $\mathrm{m}^{-2}$ (A) e número de hastes $\mathrm{m}^{-2}$ (B) de plantas de batata, cv. Macaca. Santa Maria, RS, 2004.

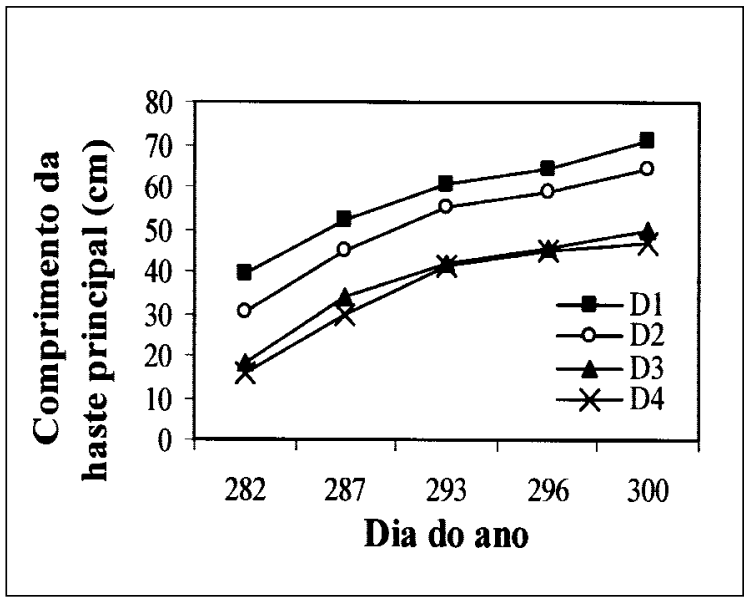

Figura 5 - Comprimento da haste principal $(\mathrm{cm})$ de plantas de batata, cv. Macaca, cultivadas em diferentes densidades. D1 $=5 \times 5, \quad \mathrm{D} 2=10 \times 10, \quad \mathrm{D} 3=15 \times 15$ e D4=20x20cm entre covas. Santa Maria, RS, 2004. comprimento da haste principal em função da densidade de plantas mostra e reforça que crescimento e desenvolvimento vegetal são processos distintos e independentes.

Os filocronos das densidades D1 e D4 (diferença de $3,7^{\circ} \mathrm{C}$ dia folha ${ }^{-1}$ ) foram utilizados para simular o aparecimento de folhas na haste principal de plantas de batata. A simulação foi feita para duas estações de cultivo, considerando-se a emergência das plantas nos dias 15/03/2003 e 15/08/2003, épocas recomendadas para o cultivo da safrinha e safra, respectivamente, na região de Santa Maria (BISOGNIN, 1996). Foi assumido que as plantas apresentavam uma folha no momento da emergência e o número final de folhas de 21, baseado no valor médio das 36 plantas avaliadas no presente estudo. Para o cultivo da safra foi estimadoum aumento de6 dias e, para a safrinha, um aumento de oito dias no período de emissão de folhas como conseqüência dos dois valores de filocrono (Figura 6). A

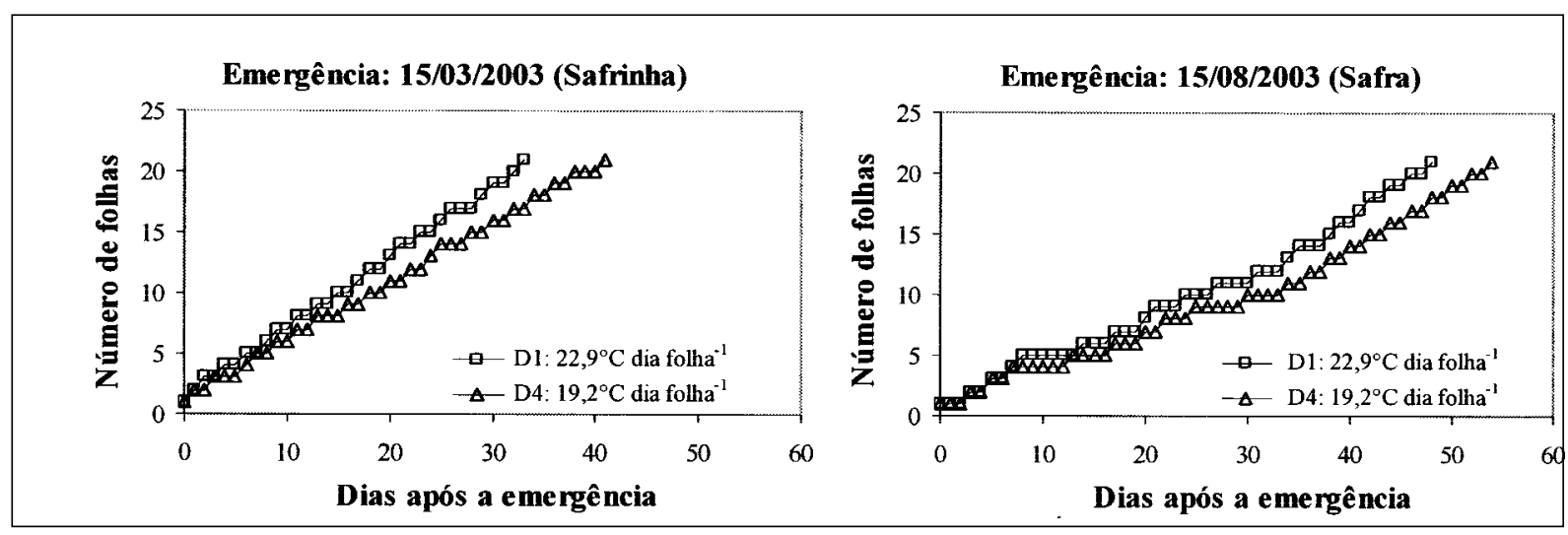

Figura 6 - Número de folhas acumuladas na haste principal de plantas de batata, cv. Macaca, simulado em duas densidades de plantio de tubérculos com dois valores de filocrono em duas épocas de cultivo. D1=5x5 e D4=20x20cm entre covas.

Ciência Rural, v.35, n.6, nov-dez, 2005. 
implicação destas simulações é que modelos de simulação do número de folhas em batata devem levar em conta a densidade de plantas.

\section{CONCLUSÃO} em batata.

A densidade de plantas afeta o filocrono

\section{AGRADECIMENTOS}

Dellai e Trentin, bolsista da Coordenação de Aperfeiçoamento de Pessoal de Nível Superior (CAPES) e do Conselho Nacional de Desenvolvimento Científico e Tecnológico (CNPq), respectivamente.

\section{REFERÊNCIAS}

ANDRIOLO, J.L.; POERSCHKE, P.R.C. Cultivo do tomateiro em substratos. Santa Maria: UFSM - Centro de Ciências Rurais, 1997. 12p. (Informe Técnico, 2).

BISOGNIN, D.A. Recomendações técnicas para o cultivo da batata no Rio Grande do Sul e Santa Catarina. Santa Maria: UFSM, 1996. 64p.

CAO, W.; TIBBITTS, T.W. Leaf emergence on potato stems in relation to thermal time. Agronomy Journal, v.87, p.474477, 1995.

FAO. FAO production yearbook 1998. Rome: FAO Statistics, 1998. Series, v.52.

FRANK, A.B.; BAUER, A. Phyllochron differences in wheat, barley and forage grasses. Crop Science, v.35, n.1, p.19-23, 1995.

HERMES, C.C. et al. Emissão de folhas de alface em função da soma térmica. Revista Brasileira de Agrometeorologia, v.9, n.2, p.269-275, 2001.

IBGE. Levantamento sistemático da produção agrícola, confronto das safras de 2003 e das estimativas para 2004. Capturado em 15 abr. 2004. Online. Disponível na Internet http://www.ibge.gov.br
JEFFERIES, R.A. et al. Thermal time as a non-destructive method of estimating tuber initiation in potatoes. Journal of Agricultural Science, v.108, p.249-252, 1987.

KIM, S.Y. et al. Hydroponic culture system for the production of seed tubers without soil. American Journal of Potato Research, v.77, n.6, p.394, 2000.

KLEPPER, B. et al. Quantitative characterization of vegetative development in small cereal grains. Agronomy Journal, v.74, n.4, p.789-792, 1982.

LOPES, C.A., BUSO, J.A. Cultivo da batata (Solanum tuberosum L.). Brasília: Embrapa Hortaliças, 1997. 36p.

MEDEIROS, C.A.B. et al. Produção de sementes pré-básicas de batata em sistemas hidropônicos. Horticultura Brasileira, v.20, n.1, p.110-114, 2002.

OLIVEIRA, C.A. da S. Potato crop growth as affected by nitrogen and plant density. Pesquisa Agropecuária Brasileira, v.35, n.5, p.939-950, 2000.

PEREIRA, A.R. et al. Evapo(transpi)ração. Piracicaba: FEALQ, 1997. 183p.

SANDS, P.J. et al. A model of the development and bulking of potatoes (Solanum tuberosum L.). I - Derivation from wellmanaged field crops. Field Crops Research, v.2, n.4, p.309331, 1979.

SAS Institute, Inc. SAS-STAT, Users guide. Version 6.0. Cary, NC: SAS Institute, 1997.

STRECK, N.A. A generalized nonlinear air temperature response function for node appearance rate in muskmelon (Cucumis melo L.). Revista Brasileira de Agrometeorologia, v.10, n.1, p.105-111, 2002.

STRECK, N.A. et al. Incorporating a chronology response into the prediction of leaf appearance rate in winter wheat. Annals of Botany, v.92, p.181-190, 2003.

VOS, J.; BIEMOND, J. Effects of nitrogen on the development and growth of the potato plant. 1. Leaf appearance, expansion growth, life spans of leaves and stem branching. Annals of Botany, v.70, n.1, p.27-35, 1992. 